\title{
Magnetic Resonance Force Detection using a Membrane Resonator
}

\author{
N. Scozzaro ${ }^{\mathrm{a}}$, W. Ruchotzke ${ }^{\mathrm{a}}$, A. Belding ${ }^{\mathrm{a}}$, J. Cardellino ${ }^{\mathrm{a}}$, E. Blomberg ${ }^{\mathrm{a}}$, B. McCullian ${ }^{\mathrm{a}}$, \\ V.P. Bhallamudi ${ }^{\mathrm{a}}$, D. V. Pelekhov ${ }^{\mathrm{a}}$, P. C. Hammel ${ }^{\mathrm{a}, *}$ \\ ${ }^{a}$ Department of Physics, The Ohio State University, Columbus, Ohio 43210, USA
}

\begin{abstract}
The availability of compact, low-cost magnetic resonance imaging instruments would further broaden the substantial impact of this technology. We report highly sensitive detection of magnetic resonance using lowstress silicon nitride $\left(\mathrm{SiN}_{x}\right)$ membranes. We use these membranes as low-loss, high-frequency mechanical oscillators and find they are able to mechanically detect spin-dependent forces with high sensitivity enabling ultrasensitive magnetic resonance detection. The high force detection sensitivity stems from their high mechanical quality factor $Q \sim 10^{6}[1,2]$ combined with the low mass of the resonator. We use this excellent mechanical force sensitivity to detect the electron spin magnetic resonance using a $\mathrm{SiN}_{x}$ membrane as a force detector. The demonstrated force sensitivity at $300 \mathrm{~K}$ is $4 \mathrm{fN} / \sqrt{\mathrm{Hz}}$, indicating a potential low temperature $(4 \mathrm{~K})$ sensitivity of $25 \mathrm{aN} / \sqrt{\mathrm{Hz}}$. Given their sensitivity, robust construction, large surface area and low cost, $\mathrm{SiN}_{x}$ membranes can potentially serve as the central component of a compact room-temperature ESR and NMR instrument having spatial resolution superior to conventional approaches.
\end{abstract}

Keywords: Silicon-nitride, Membrane, MRFM, Cyclic-saturation, ESR, MRI, Quality-factor

\section{Introduction}

Magnetic resonance is a powerful tool that has had substantial impact on the fields of medicine, chemistry, and physics. Modern nuclear magnetic resonance (NMR) and magnetic resonance imaging (MRI) apparatuses utilize technology that has benefited from six decades of development, but their impact could be amplified in some settings by reducing their cost and physical size. Higher sensitivity can also enable enhanced spatial resolution in imaging applications.

Magnetic resonance force microscopy (MRFM) is based on mechanical detection of magnetic resonance signals. It has demonstrated imaging resolution far beyond that of the best inductive-based MRI [3, 4, 5, 6], achieving nuclear-spin resolution better than ten nanometers $[7,8]$. The central component of MRFM is a mechanical resonator that is used to sensitively measure the force of interaction between the sample containing electron or nuclear

\footnotetext{
* Corresponding author

Email address: Hammel@physics.osu.edu (P. C. Hammel)

Preprint submitted to Elsevier
}

spins, and a probe magnet with a strong field gradient. Depending on the experimental configuration, either the sample or the probe magnet is placed directly on the resonator $[7,9]$ while the other component is in a fixed position in close proximity. The force of the probe-sample interaction is measured by detecting the displacement of the resonator via optical interferometry. Thermal force noise as low as $0.82 \mathrm{aN} / \sqrt{\mathrm{Hz}}$ was achieved [10] using MRFM, culminating in an experiment that demonstrated single electron spin detection [9]. High sensitivity allows smaller volumes to be detected, hence enablihg high spatial resolution. At present such a high sensitivity has been achieved by using ultrasoft cantilevers with spring constants $k$ as low as $110 \mu \mathrm{N} / \mathrm{m}$ [9]. While such cantilevers deliver exceptional force sensitivity, their use presents challenges: they are very fragile, sample preparation in the sample-on-cantilever geometry is difficult due to its small size, and such cantilevers are not commercially available.

Here we demonstrate that $\operatorname{SiN}_{x}$ membranes present a viable alternative to ultrasoft cantilevers as sensitive force detectors for MRFM applications. Such membranes exhibit a number of at-

July 21, 2016 
tractive properties including high sensitivity, robust mechanical properties, commercial availability, low cost, and a large surface area. The force noise of membranes can be as low as $8 \mathrm{aN} / \sqrt{\mathrm{Hz}}$ $[2,11,12]$, which is within an order of magnitude of the highest sensitivity demonstrated so far for an ultrasoft cantilever [10]. While less sensitive, the membrane is much less fragile because it is surrounded on all sides, unlike a cantilever supported only at one end. As a result, the membrane is much less susceptible to bending and twisting which otherwise can be detrimental for interferometric displacement detection. Samples can be quickly prepared on membranes utilizing similar sample preparation techniques as transmission electron microscopy (TEM), including application to the membrane by micropipette, or immersion in fluid on a glass slide. Finally, with their large surface area, membranes can accommodate a wider range of sample sizes and provide a larger target for interferometry than ultrasoft cantilevers.

One further advantage of membranes is their high natural frequency. The fundamental frequency of membranes can be in the $\mathrm{MHz}$ range, which enables resolving faster spin dynamics, and opens the door to new experiments. For example, since the Larmor frequency of nuclear spins in low field is also in the $\mathrm{MHz}$ range, there is the possibility of matching the membrane's mechanical resonance with the spins nuclear magnetic resonance frequency. This matching could overcome the current restriction to measuring only the $z$-component of the magnetization and allow force detection of its transverse component. This enticing possibility would provide MRFM access to the powerful array of imaging tools developed for pulsed NMR. Transverse detection of Larmor precession, known as directdetection or "spin precession imaging" [13, 14], could furthermore be accomplished without the necessity of an RF-generator. As the membrane oscillates, the spin sample on the membrane is physically displaced in the presence of a strong field gradient, which naturally generates the large oscillating magnetic field needed to excite the magnetic resonance signal. This innovation would aid in simplifying the MRFM apparatus. The apparatus could thus be reduced to three main components: a membrane, optical fiber-based displacement detection, and a magnetic particle on a translation stage as depicted in figure 1.

\section{Experiment}

\subsection{Experimental details and setup}

The MRFM experiment we report is performed by creating an oscillating force on a sample placed in the center of the membrane. The oscillating force is generated by modulating the sample magnetization using magnetic resonance, in the presence of a field gradient. The force drives the membrane at its natural frequency to an amplitude $A=F Q / k$, where $F$ is the force, $k$ is the spring constant, and $Q$ is the quality factor of the membrane. We use the cyclic-saturation resonance protocol $[15,16]$ to measure a small particle of diphenyl picrahydrazyl (DPPH) on a membrane. DPPH is a well-known organic molecule that exhibits a electron paramagnetic resonance (EPR) signal.

The magnetic resonance signal is detected by measuring the displacement of the $\mathrm{SiN}_{x}$ membrane by means of a fiber optic interferometer, aligned as shown in figure 1 . The interferometer uses 1550 $\mathrm{nm}$ laser light and is focused down to a $10 \mu \mathrm{m}$ spot adjacent to the DPPH particle. The optical power incident on the membrane is about $80 \mu \mathrm{W}$. A 2.5 turn, $350 \mu \mathrm{m}$ diameter copper resonance coil generates $B_{1}$, the $\mathrm{RF}$ field. The coil is centered on the membrane and is stub-tuned to a frequency $\omega_{0} / 2 \pi=3.010 \mathrm{GHz}$, setting the magnetic resonance condition $\omega_{0} / \gamma=1070 \mathrm{G}$, where $\gamma$ is the electron gyromagnetic ratio. With an input power of $100 \mathrm{~mW}$, the coil produces $B_{1}=4.7 \mathrm{G}$. On the opposite side of the membrane, a two-axis piezoelectric stage [17] positions a rectangular NeFeB magnet, which provides both the polarizing magnetic field $\left(B_{0}\right)$ and a field gradient of $G=0.2 \mathrm{G} / \mu \mathrm{m}$. The instrument operates at a pressure of $10^{-6}$ torr.

Using the coordinate system in figure 1 , the force on the electrons in the DPPH is given by $F=\mu_{z} \frac{\mathrm{d} B_{z}}{\mathrm{~d} x}$, where $\mu_{z}=M_{z} V$, and $V$ is the volume of the DPPH particle. The Bloch equations give the magnetization

$$
M_{z}=M_{0}\left(1-\frac{\gamma^{2} B_{1}^{2} \tau^{2}}{1+\left(\gamma B_{0}-\omega\right)^{2} \tau^{2}+\gamma^{2} B_{1}^{2} \tau^{2}}\right)
$$

where $M_{0}=\frac{\chi_{0} B_{0}}{\mu_{0}}$ is the thermal equilibrium magnetization, $B_{0}^{\mu_{0}}$ is the magnetic field in the $z$ direction generated by the permanent magnet, $\chi_{0}=$ $2.5 \times 10^{-5}$ is the susceptibility of DPPH, $\mu_{0}$ is the permeability of vacuum, $\tau=62 \mathrm{~ns}$ is the spin relaxation time, and $\frac{\gamma}{2 \pi}=28 \mathrm{GHz} / \mathrm{T}$ is the electron gyromagnetic ratio. To create the oscillating force, 


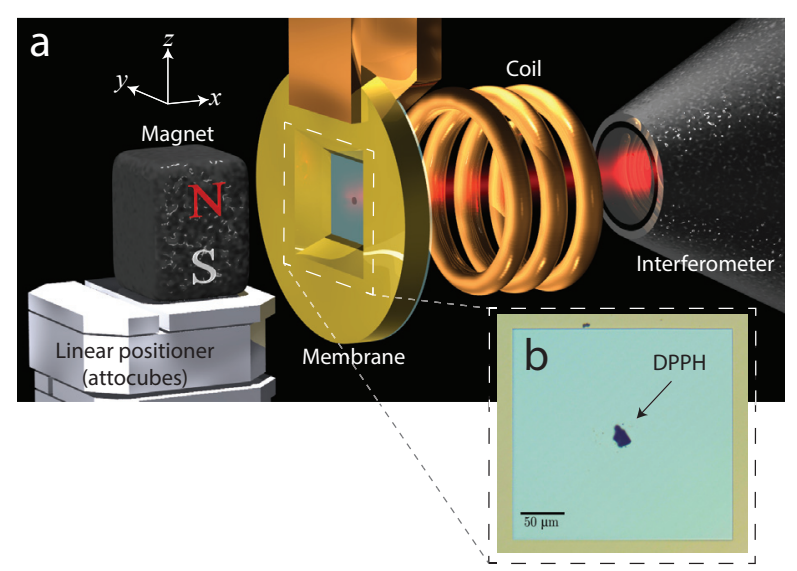

Figure 1: Experimental schematic: (a) Schematic of the experimental setup. From the left, the interferometer laser passes through the copper coil and is incident on the membrane. A $20 \mu \mathrm{m}$ particle of DPPH is placed in the center of the membrane. A permanent magnet produces both the polarizing field $B_{0}$ and a magnetic field gradient, which generates a force on the electron spins in the DPPH. (b) Photomicrograph of the $30 \mathrm{~nm}$ thick, $250 \mu \mathrm{m}$ side-length silicon nitride membrane (inner turquoise square), surrounded by the silicon support. In the center of the membrane is the piece of DPPH is attached using a small amount of G1 epoxy. The bare membrane exhibits a frequency of $1.35 \mathrm{MHz}$, and the loaded membrane exhibits a frequency of $644 \mathrm{kHz}$.

we induce an oscillating moment at the membrane frequency by modulating the frequency $\omega$ of $B_{1}$ such that $\omega(t)=\omega_{0}+\Omega \sin \left(2 \pi f_{c} t\right)$, where $f_{c}$ is the membrane frequency. This results in a time-varying magnetization $M_{z}(t)$ whose Fourier component $M_{1}$ at the membrane frequency is given by $M_{1}=\Omega \frac{\partial M_{z}}{\partial \omega}$ [15]. The derivative leads to a bipolar line shape of the force as a function of $B_{0}$.

The magnetic resonance signal is measured by varying the position of the permanent magnet and hence the magnitude of the applied magnetic field experienced by the sample on the membrane. The region of the magnet's field where the resonance condition is satisfied $(B=1070 \mathrm{G})$ is referred to as the "resonant slice," the thickness of which is $\Delta B / G \sim 10 \mu \mathrm{m}$, where $\Delta B \sim 2 \mathrm{G}$ is the linewidth of DPPH. Due to the gradient, each spin in the resonant slice experiences a slightly different field, so the force as a function of magnet position is an integral as described in reference [16]; see the appendix.

\subsection{Force noise}

The central component of the MRFM apparatus is a sensitive mechanical oscillator which is employed as a force detector whose force sensitivity is limited by thermal force noise $S_{F}$ given by

$$
S_{F}^{1 / 2}=\left(\frac{2 k k_{B} T}{\pi Q f_{c}}\right)^{1 / 2},
$$

where $k$ is the spring constant, $k_{b}$ is the Boltzmann constant, $T$ is the temperature, $Q$ is the quality factor, and $f_{c}$ is the natural frequency. It is illuminating to cast equation 2 in terms of intrinsic membrane parameters such as thickness, side length, tensile stress, and density; $t, L, \sigma$, and $\rho$, respectively. The frequency is given by $f_{0}=\sqrt{\frac{\sigma}{2 \rho L^{2}}}$, and the spring constant is given by $k=\frac{\pi^{2} \sigma t}{2}$ [18], yielding

$$
\begin{aligned}
S_{F}^{1 / 2}= & (2 \rho \sigma)^{1 / 4}\left(\frac{\pi L t k_{B} T}{Q}\right)^{1 / 2} \\
& \sim(\rho \sigma)^{1 / 4}\left(\frac{t^{3} k_{B} T}{L}\right)^{1 / 2},
\end{aligned}
$$

where in the last step we further simplified the expression using the experimentally observed relation $[1,19]$ that quality factor goes as $Q \sim(L / t)^{2}$ for $L / t<10^{5}$. Equation (3) shows that to minimize the thermal force noise, it is desirable to have a low stress, low density material, large side length, and most critically, a very thin membrane.

To this end, we use a $30 \mathrm{~nm}$ thick, $0.25 \mathrm{~mm}$ side length membrane for our experiments $(L / t=$ $\left.8 \times 10^{3}\right)$. The membranes we used are made of lowstress $\operatorname{SiN}_{x}$ [20], and exhibit a natural frequency of $f_{c}=1.35 \mathrm{MHz}$. Given the density of low-stress silicon nitride, $\rho=3.1 \mathrm{~g} / \mathrm{cm}^{3}$, this corresponds to a stress of $\sigma=654 \mathrm{MPa}$, and a spring constant of $k \sim 100 \mathrm{~N} / \mathrm{m}$. After attaching a DPPH particle to the center of the membrane with epoxy, the frequency of the membrane decreased to roughly 644 $\mathrm{kHz}$. This corresponds to an increase in mass of $4.65 \times 10^{-12} \mathrm{~kg}$, or a particle diameter of $19 \mu \mathrm{m}$, consistent with the optical image of the membrane particle in figure $1 \mathrm{~b}$.

\subsubsection{Membrane characterization}

We measure the displacement of the membrane using a fiber-optic interferometer and we fit the thermal peak and background (figure 2a) using the equation

$$
S_{x}^{1 / 2}=\left(\frac{f_{0} k_{B} T}{2 \pi k Q\left(\left(f-f_{0}\right)^{2}+\left(\frac{f_{0}}{2 Q}\right)^{2}\right)}+S_{\mathrm{bgd}}\right)^{1 / 2}
$$


a)

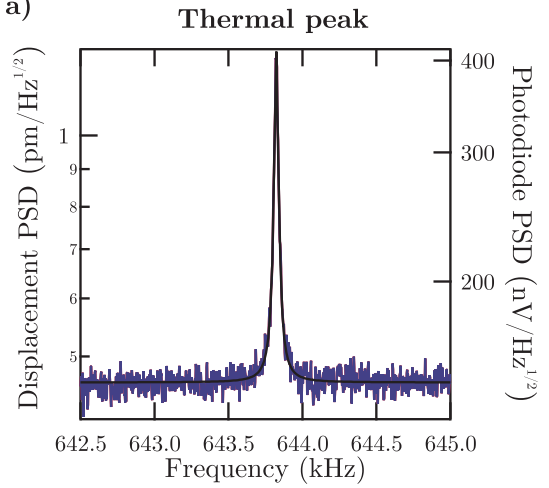

b) Quality factor from ringdowns

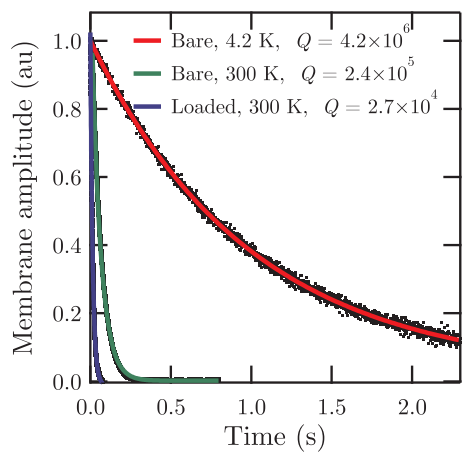

c) Frequency vs temperature

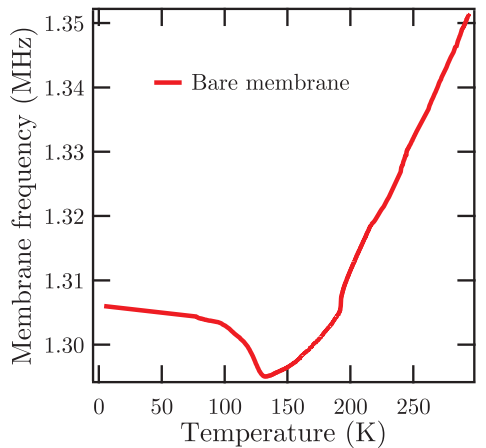

Figure 2: Characterization of the membrane signal (a) Thermal peak of the DPPH-loaded membrane measured at 300 K. The membrane frequency dropped from $1.35 \mathrm{MHz}$ when bare to $\sim 644 \mathrm{kHz}$ when loaded. The peak is fit to equation (4) by fixing $T=300 \mathrm{~K}$, and $Q=27,000$ from the ringdown measurement in panel (b). The free parameters are the spring constant, the center frequency, and the white background interferometer noise, yielding $k \sim 75 \mathrm{~N} / \mathrm{m}, f_{0}=643.8 \mathrm{kHz}, S_{b g d}^{1 / 2}=0.46$ $\mathrm{pm} / \sqrt{\mathrm{Hz}}$. (b) Membrane ringdown measurements under 3 conditions: (1) Bare membrane at $4 \mathrm{~K}$, (2) Bare membrane at 300 $\mathrm{K}$, and (3) DPPH-loaded membrane at $300 \mathrm{~K}$. We find that cryogenic temperatures increase the quality factor of the membrane by over an order of magnitude, from $2.4 \times 10^{5}$ to $4.2 \times 10^{6}$. On the other hand, loading the membrane with a particle of DPPH drastically reduces the quality factor down to $2.7 \times 10^{4}$. (c) The membranes frequency as a function of temperature. This non-monotonic behavior is likely related to the differential thermal expansion coefficient between the silicon nitride thin film and the silicon substrate $[21,22]$.

where $S_{b g d}$ is the displacement noise floor of our interferometer in units of $\mathrm{m}^{2} / \mathrm{Hz}$. Fixing $T=300$ $\mathrm{K}$ and $Q=27,000$ from a ringdown measurement (figure $2 \mathrm{~b}$ ), the fit yields $f_{0}=643,800 \mathrm{~Hz}$, $S_{b g d}^{1 / 2}=0.46 \mathrm{pm} / \sqrt{\mathrm{Hz}}$, and $k=75 \mathrm{~N} / \mathrm{m}$, in reasonable agreement with the spring constant of $\sim 100$ $\mathrm{N} / \mathrm{m}$ predicted based on the loaded frequency of the membrane. From the equipartition theorem the membrane's thermal RMS amplitude is $x_{r m s}=$ $\sqrt{\frac{k_{B} T}{k}}=7.4 \mathrm{pm}$.

We perform ringdown measurements (figure $2 \mathrm{~b}$ ) to extract the membrane's quality factor by driving the membrane, ceasing the drive, then measuring the amplitude decay with a lock-in amplifier. We fit the amplitude decay to an exponential, $A e^{-\frac{t}{\tau_{c}}}$, and use $Q=\pi f_{0} \tau_{c}$ to extract $Q$. The bare membrane exhibited a quality factor of $2.4 \times 10^{5}$ at room temperature, which increased to $4.2 \times 10^{6}$ upon cooling the system to low temperature. The impressively large $Q$ at low temperature $(4 \mathrm{~K})$ is in agreement with other measurements $[1,2]$. Loading the membrane with the DPPH particle significantly reduced the quality factor to $Q=2.7 \times 10^{4}$. Utilizing equation (2) and the measured quality factors, we find a room temperature thermal force noise of $4 \mathrm{fN} / \sqrt{\mathrm{Hz}}$ for the loaded membrane, and $25 \mathrm{aN} / \sqrt{\mathrm{Hz}}$ for the bare membrane at low temperature. While we have found that adding a large particle will drastically reduce the quality factor of the membrane, thereby making the membrane less sensitive, we have also placed particles on the membrane $(\sim 1 \mu \mathrm{m})$ small enough that they did not affect the quality factor.

As the bare membrane is cooled to cryogenic temperatures (figure 2c), we observe that the frequency decreases for temperatures between $300 \mathrm{~K}$ and 130 $\mathrm{K}$, then increases between $130 \mathrm{~K}$ and $4 \mathrm{~K}$. We attribute this non-monotonic behavior to the differential coefficient of thermal expansion between the $\mathrm{SiN}_{x}$ thin films and the silicon substrate. For high temperature $\mathrm{SiN}_{x}$ thin film deposition on silicon, the coefficient of thermal expansion for silicon is greater than that of $\mathrm{SiN}_{x}$ which results in a decrease in the stress of the membrane as it cools [21, 22], and correspondingly the membrane frequency decreases. As the temperature continues to decrease past $130 \mathrm{~K}$ the coefficients cross, reversing the effect: the stress increases, and membrane frequency increases.

\section{Results}

Figure 3 shows the measured DPPH magnetic resonance signal as a function of microwave power. The experimental parameters are $\omega_{0} / 2 \pi=3.010$ 


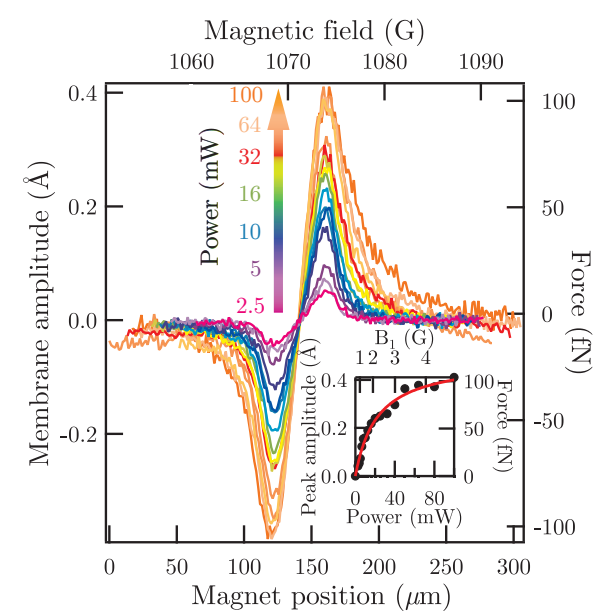

Figure 3: Cyclic saturation power dependence Microwave power is increased from $2.5 \mathrm{~mW}$ to a maximum of $100 \mathrm{~mW}$, corresponding to a $B_{1}$ of $4.7 \mathrm{G}$. The signal grows with increasing microwave power, peaking with an amplitude of $0.4 \AA$ corresponding to a force of $100 \mathrm{fN}$. Each curve is fit with equation 5 , and the peak force is extracted and plot in the inset. The overlaid curve in the inset is from the maximum value of equation 5 as a function of microwave power.

GHz, $\Omega / 2 \pi=10 \mathrm{MHz}, T=300 \mathrm{~K}$. We take multiple measurements at each point, and use more averaging for smaller powers. The high power scans (150 points) took about 40 minutes, and the lower power sweeps took up to 2 hours. We fit each curve with equation (5) (appendix) and extract the peak forces, which are shown in the inset. The magnetic force drives the membrane to a maximum vibration amplitude of 0.4 angstroms as shown on the right axis for a power of $100 \mathrm{~mW}$, corresponding to $B_{1}=4.7 \mathrm{G}$. The gradient we extract is approximately $G=0.2 \mathrm{G} / \mu \mathrm{m}$, and the force corresponds to a polarized moment of $\mu_{z}=10^{-14} \mathrm{~J} / \mathrm{T}$, or $10^{9}$ electron spins.

We also measure higher harmonic spin signals (figure 5) which have been shown to reduce spurious noise [15], and provide additional confirmation of the origin of the signal. Due to the nonlinearity of equation 1 with respect to RF modulation, magnetic resonance signals are generated not just at the modulation frequency $\omega_{m}$, but also at integer multiples: $2 \omega_{m}, 3 \omega_{m}$, etc. We measure these higher harmonic signals by setting the microwave modulation rate at half the membranes frequency $\omega_{m}=\left(2 \pi f_{c}\right) / 2$, or one-third, etc., resulting in a signal at the membrane's frequency which we de-

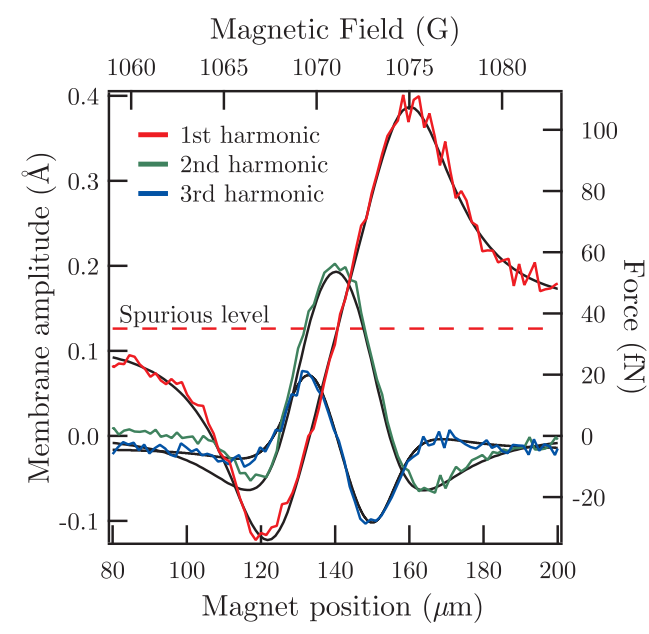

Figure 4: Higher harmonic data Measurement of the first three harmonic spin signals with a power of $32 \mathrm{~mW}$. The first harmonic data (red) is identical to that shown in figure 3 , and is provided for reference.The second harmonic and third harmonic spin signals are the green and blue curves respectively. Second and third harmonic measurements are performed by modulating the microwave frequency at half and one third the membrane frequency, respectively, while monitoring the membrane amplitude at the membranes natural frequency. The first, second, and third harmonic signals are proportional to the first, second, and third derivatives of the magnetization, respectively.

tect. The first three harmonic signals are given by $M_{1}=\Omega \frac{\partial M_{z}}{\partial \omega}, M_{2}=\frac{\Omega^{2}}{4} \frac{\partial^{2} M_{z}}{\partial \omega^{2}}, M_{3}=\frac{\Omega^{3}}{24} \frac{\partial^{3} M_{z}}{\partial \omega^{3}}$; each additional harmonic is one higher derivative. For modulation at the membrane frequency and $32 \mathrm{~mW}$ of microwave power, spurious coupling appears as a constant oscillation amplitude offset of $0.1 \AA$ in our in-phase lock-in channel, on top of which rides the $0.3 \AA$ spin signal. We subtract this background from the data in figure 3 . In contrast, spurious coupling is nearly eliminated using modulation away from the membranes frequency, and no background subtraction is necessary in figure 5 .

\section{Discussion}

We now address the potential for detection of nuclear spins with membranes. Detection of nuclear moments is more difficult than detecting electrons for two reasons: $(1)$ a very large $B_{1}(>100 \mathrm{G})$ is needed to adiabatically invert nuclear spins at the membrane frequency, and (2) the moment of nuclear spins is much smaller than that of electrons.

When the relaxation time of spins is longer than the period of the mechanical oscillator, cyclic sat- 
uration cannot be used to generate an oscillating force for MRFM detection. Instead, adiabatic inversion of spins is necessary, which requires $B_{1} \gg$ $\sqrt{\frac{4 f_{c} \Omega}{\gamma^{2}}}$, where $\Omega$ is the depth of frequency modulation. The MRFM signal grows linearly with $\Omega$, so it cannot be made arbitrarily small. Since the frequency of the membrane is very large, this means that a very large $B_{1}$ is required, over $100 \mathrm{G}$. While it is challenging to engineer such strong $B_{1}$, Nichol et. al used a $240 \mathrm{~nm}$ wide, $100 \mathrm{~nm}$ thick constriction in a silver wire to achieve a $B_{1}$ of $88 \mathrm{G}$ for MRFM detection [8, 23].

The minimum number of spins $n$ that can be measured is set by Curie's law and the thermal force noise of the membrane [24], $n=\frac{\sqrt{2} S_{F} \Delta \nu k_{B} T}{\mu_{N}^{2} G}$, where $\mu$ is the nuclear or electron spin moment, and $\Delta \nu$ is the detection bandwidth. Given that the electron has a moment 660 times larger than that of a nuclear spin such as a proton, a significantly smaller number of electron spins are required to achieve the same signal as nuclear spins. For our membrane under the conditions presented in this paper, the minimum number of total protons we would be able to detect at room temperature is $5.5 \times 10^{16}$ protons, corresponding to a voxel size of $475 \mu \mathrm{m}$ (assuming a spin density of $5.1 \times 10^{28}$ protons $/ \mathrm{m}^{3}$ ). However, with experimentally accessible parameters such as a gradient of $40 \mathrm{G} / \mathrm{nm}$ [7], a polarizing field of $5 \mathrm{~T}$, and operating at low temperature, the membrane can potentially be used to detect $3.7 \times 10^{4}$ spins corresponding to a voxel size of $40 \mathrm{~nm}$.

Current MRFM measures the longitudinal component of the magnetization. An alternative approach is to measure the transverse component of the precessing magnetization. Transverse detection has the challenge that it requires high frequency detection, which a membrane can provide. An additional advantage is that the oscillation of the membrane in a gradient field can directly provide the AC transverse field needed for magnetic resonance. This approach involves placing a micromagnet such that it creates an inhomogeneous transverse field at the position of the spin sample on the membrane. If the membrane is driven to a peak amplitude of $x_{\mathrm{pk}}$ the spin sample will experience a transverse oscillating magnetic field $B_{1}=G x_{\mathrm{pk}} \sin \omega_{0} t$, where $G$ is the magnetic field gradient perpendicular to the membrane and $\omega_{0}$ is the membrane frequency. For example, with a typical driven membrane amplitude of $150 \mathrm{~nm}$, a gradient of $1 \mathrm{G} / \mathrm{nm}$ would produce $B_{1}=150 \mathrm{G}$. The nuclear magnetic reso- nance condition can be satisfied by matching the nuclear Larmor frequency to the membrane's natural frequency through application of the appropriate external field. Sensitive nuclear spin detection can potentially be implemented by measuring the mechanical damping of the membrane as the field is swept through resonance in a mechanical analog to SQUID-based induction measurements of transverse spin noise $[25,26]$.

\section{Conclusion}

We have provided the first demonstration of magnetic resonance force microscopy using a membrane resonator. We measured the signal from a $20 \mu \mathrm{m}$ DPPH particle placed on a membrane. The membrane exhibited a room temperature sensitivity of 4 $\mathrm{fN} / \sqrt{\mathrm{Hz}}$, and a potential low temperature sensitivity of $25 \mathrm{aN} / \sqrt{\mathrm{Hz}}$. Membranes are a practical mechanical resonator for use in MRFM because they are commercially available, low cost, and have high sensitivity. Furthermore they are versatile in that they have a large surface area, high resonant frequency, and can be used in the fields of quantum optomechanics, TEM, and MRFM. Given these properties, membranes are a compelling candidate for a number of applications such as a compact detector that can be used to identify or image nuclear spins in microscopic samples.

\section{Acknowledgments}

The research presented in this Article was supported by the Army Research Office (grant no. W911NF-09-1-0147), and the Center for Emergent Materials (CEM), an NSF-funded MRSEC through grant DMR-1420451. Technical support was provided by the NanoSystems Laboratory at The Ohio State University.

\section{Appendix}

\subsection{Fitting Cyclic Saturation Signals}

To fit the cyclic saturation data of figure 3, we calculate the net force acting on the DPPH particle following reference [16]. Frequency modulation causes the resonant slice to oscillate spatially. The resonant slice is $50 \mu \mathrm{m}$ in thickness, and the particle is $20 \mu \mathrm{m}$ in size. As the magnet approaches the particle, the leading edge of the slice begins to cyclically enter and exit the particle. This creates a 
force which resonantly drives the membrane. Once the resonant slice is fully inside the particle, there is no net force because spins on either side of the resonant slice create oscillating forces with opposite phase, yielding cancellation of the net force. As the resonant slice exits the other side of the particle, a force is created 180 degrees out of phase with the drive, which the lock-in records as a negative value.

We model the magnet as a sphere that generates a dipole field $B_{0}=\frac{\mu_{0}}{4 \pi} \frac{m}{\left(x-x_{\text {magnet }}\right)^{3}}$ and corresponding gradient, $G=\frac{-3 \mu_{0}}{4 \pi} \frac{m}{\left(x-x_{\text {magnet }}\right)^{4}}$, where $m=\frac{4}{3} \pi R^{3} M, M=1.06 \times 10^{6}$ is the magnetization of the NeFeB magnet, and $R \sim 1 \mathrm{~cm}$ is the radius of the magnet. We assume the DPPH particle has roughly a constant cross sectional area $A$. We thus have an equation for the total force on the DPPH particle, which we use to fit the data in figure 3,

$$
\begin{aligned}
& F\left(x_{\text {magnet }}\right)=\int_{x_{\text {start }}}^{x_{\text {end }}} G M_{1} A \mathrm{~d} x= \\
& \frac{A B_{1}^{2} \gamma M_{0} \tau^{2} \Omega}{1+B_{1}^{2} \gamma^{2} \tau^{2}+\tau^{2}\left(\omega_{0}-\frac{\mu_{0} \gamma M R^{3}}{3\left(x_{\text {magnet }}-x_{\text {start }}\right)^{2}}\right)^{2}} \\
& -\frac{A B_{1}^{2} \gamma M_{0} \tau^{2} \Omega}{1+B_{1}^{2} \gamma^{2} \tau^{2}+\tau^{2}\left(\omega_{0}-\frac{\mu_{0} \gamma M R^{3}}{3\left(x_{\text {magnet }}-x_{\text {end }}\right)^{2}}\right)^{2}}
\end{aligned}
$$

where $x_{\text {start }}$ and $x_{\text {end }}$ denote the position of the DPPH particle, and $x_{\text {magnet }}$ is the magnet's position. As the resonant slice passes through the DPPH particle, we measure the cyclic saturation signal shown in figure 3. For the second and third harmonics we replace $M_{1}$ with $M_{2}$ and $M_{3}$ in equation 5 and solve for similar expressions, which we use to fit the data in figure 5 .

\subsection{Duffing Oscillator Behavior}

Using a piezoelectric element we can sinusoidally drive the membrane to large amplitudes as shown in figure 2c. Due to a cubic term in the spring response of the membrane, $F=k x+\beta x^{3}$, the driven equation of motion is non-linear and is given by $\ddot{x}+\left(\frac{\omega_{c}}{Q}\right) \dot{x}+\omega_{c}^{2}+\beta x^{3}=F_{0} \sin \omega_{d} t[27]$, where $\omega_{d}$ is the drive frequency and $\beta$ is a parameter that describes the strength of the nonlinearity. The result is a non-Lorentzian Duffing oscillator frequency dependence of the membrane response. The shape of the signal can be understood in that the membrane effectively becomes stiffer for large amplitudes of oscillation, causing the natural frequency of the membrane to increase. We work at sufficiently low

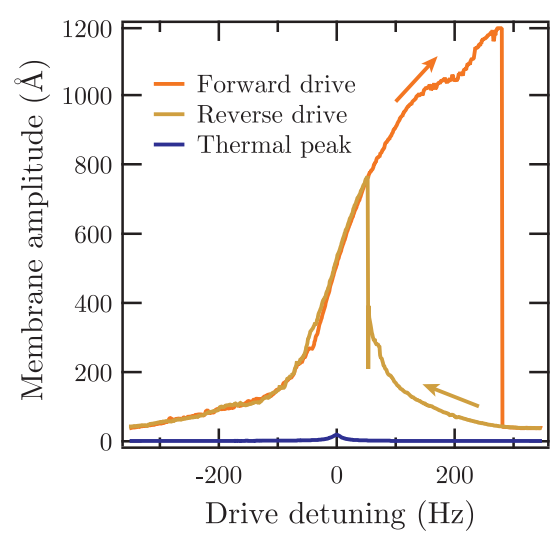

Figure 5: Duffing-oscillator reponse Driving the membrane to large amplitudes shows the well-known Duffingoscillator response, which results from a cubic term in addition to the linear term in the spring constant. The nonlinearity results in an increase in resonance frequency for large drive amplitudes, as well as causing the sharp discontinuities, and the hysteresis in scanning forward or reverse.

drive amplitudes that our experiment is in the linear regime.

[1] Chakram, S., Patil, Y. S., Chang, L. \& Vengalattore, M. Dissipation in ultrahigh quality factor sin membrane resonators. Phys. Rev. Lett. 112, 127201 (2014). URL http://link.aps.org/doi/10. 1103/PhysRevLett.112.127201.

[2] Zwickl, B. M. et al. High quality mechanical and optical properties of commercial silicon nitride membranes. Applied Physics Letters 92, (2008). URL http://scitation.aip.org/content/ aip/journal/apl/92/10/10.1063/1.2884191.

[3] Ciobanu, L., Seeber, D. \& Pennington, C. 3d mr microscopy with resolution $3.7 \mu \mathrm{m}$ by $3.3 \mu \mathrm{m}$ by $3.3 \mu \mathrm{m}$. Journal of Magnetic Resonance 158, 178 - 182 (2002). URL http://www.sciencedirect.com/ science/article/pii/S109078070200071X.

[4] Lee, S.-C. et al. One micrometer resolution $\mathrm{nmr}$ microscopy. Journal of Magnetic Resonance 150, 207 - 213 (2001). URL http://www.sciencedirect.com/ science/article/pii/S1090780701923195.

[5] Glover, P. \& Mansfield, S. P. Limits to magnetic resonance microscopy. Reports on Progress in Physics 65, 1489 (2002). URL http://stacks.iop.org/0034-4885/ $65 / i=10 / a=203$.

[6] Moore, E. \& Tycko, R. Micron-scale magnetic resonance imaging of both liquids and solids. Journal of Magnetic Resonance 260, 1 - 9 (2015). URL http://www.sciencedirect.com/science/article/ pii/S1090780715001986.

[7] Degen, C. L., Poggio, M., Mamin, H. J., Rettner, C. T. \& Rugar, D. Nanoscale magnetic resonance imaging. Proceedings of the National Academy of Sciences 106, 1313-1317 (2009). URL http:// www. pnas.org/content/106/5/1313.abstract. http: //www.pnas.org/content/106/5/1313.full.pdf.

[8] Nichol, J. M., Hemesath, E. R., Lauhon, L. J. \& 
Budakian, R. Nanomechanical detection of nuclear magnetic resonance using a silicon nanowire oscillator. Phys. Rev. B 85, 054414 (2012). URL http: //link.aps.org/doi/10.1103/PhysRevB.85.054414.

[9] Rugar, D., Budakian, R., Mamin, H. J. \& Chui, B. W. Single spin detection by magnetic resonance force microscopy. Nature 430, 329-332 (2004). URL http: //dx.doi.org/10.1038/nature02658.

[10] Mamin, H. J. \& Rugar, D. Sub-attonewton force detection at millikelvin temperatures. Applied Physics Letters 79, 3358-3360 (2001). URL http://scitation.aip.org/content/aip/journal/ apl/79/20/10.1063/1.1418256.

[11] Norte, R. A., Moura, J. P. \& Gröblacher, S. Mechanical resonators for quantum optomechanics experiments at room temperature. Phys. Rev. Lett. 116, 147202 (2016). URL http://link.aps.org/doi/10. 1103/PhysRevLett.116.147202.

[12] Reinhardt, C., Müller, T., Bourassa, A. \& Sankey, J. C. Ultralow-noise sin trampoline resonators for sensing and optomechanics. Phys. Rev. X 6, 021001 (2016). URL http://link.aps.org/doi/10.1103/PhysRevX.6. 021001.

[13] Sidles, J. A. et al. Magnetic resonance force microscopy. Rev. Mod. Phys. 67, 249-265 (1995). URL http:// link.aps.org/doi/10.1103/RevModPhys.67.249.

[14] Sidles, J. A., Garbini, J. L. \& Drobny, G. P. The theory of oscillator-coupled magnetic resonance with potential applications to molecular imaging. Review of Scientific Instruments 63, 3881-3899 (1992). URL http://scitation.aip.org/content/ aip/journal/rsi/63/8/10.1063/1.1143837.

[15] Rugar, D., Yannoni, C. S. \& Sidles, J. A. Mechanical detection of magnetic resonance. Nature 360, 563-566 (1992). URL http://dx.doi.org/10.1038/360563a0.

[16] Wago, K. et al. Magnetic resonance force detection and spectroscopy of electron spins in phosphorusdoped silicon. Review of Scientific Instruments $\mathbf{6 8}$, 1823-1826 (1997). URL http://scitation.aip.org/ content/aip/journal/rsi/68/4/10.1063/1.1147967.

[17] Attocube Systems AG. URL http://http://www. attocube.com/.

[18] Zwickl, B. M., Yang, C., Sankey, J., Jayich, A. \& Harris, J. Progress Toward Observation of Radiation Pressure Shot Noise. Ph.D. thesis, Yale University (2011).

[19] Unterreithmeier, Q. P., Faust, T. \& Kotthaus, J. P. Damping of nanomechanical resonators. Phys. Rev. Lett. 105, 027205 (2010). URL http://link.aps.org/ doi/10.1103/PhysRevLett.105.027205.

[20] Structure Probe Inc. URL https://www.2spi.com/.

[21] Martyniuk, M., Antoszewski, J., Musca, C. A., Dell, J. M. \& Faraone, L. Environmental stability and cryogenic thermal cycling of low-temperature plasmadeposited silicon nitride thin films. Journal of Applied Physics 99 (2006). URL http://scitation.aip.org/ content/aip/journal/jap/99/5/10.1063/1.2179969.

[22] Swenson, C. A. Recommended values for the thermal expansivity of silicon from 0 to $1000 \mathrm{k}$. Journal of Physical and Chemical Reference Data 12, 179182 (1983). URL http://scitation.aip.org/content/ aip/journal/jpcrd/12/2/10.1063/1.555681.

[23] Nichol, J. Nanoscale magnetic resonance imaging using silicon nanowire oscillators. Ph.D. thesis, University of Illinois at Urbana-Champaign (2014).

[24] Rugar, D. et al. Force detection of nuclear magnetic resonance. Science 264, 10 (1994).

[25] Sleator, T., Hahn, E. L., Hilbert, C. \& Clarke, J. Nuclear-spin noise. Phys. Rev. Lett. 55, 17421745 (1985). URL http://link.aps.org/doi/10.1103/ PhysRevLett.55.1742.

[26] Sleator, T., Hahn, E. L., Hilbert, C. \& Clarke, J. Nuclear-spin noise and spontaneous emission. Phys. Rev. B 36, 1969-1980 (1987). URL http://link.aps. org/doi/10.1103/PhysRevB.36.1969.

[27] Landau, L. \& Lifshitz, E. Classical mechanics (Pergamon Press, Oxford, 1960). 


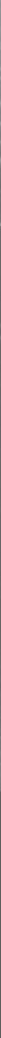

